\title{
The Outcome of the Axillofemoral Bypass: A Retrospective Analysis of 45 Patients
}

\author{
Marjolein H. Liedenbaum · Froukje J. Verdam • \\ David Spelt · Hans G. W. de Groot • \\ Jan van der Waal $\cdot$ Lijckle van der Laan
}

Published online: 22 August 2009

(C) The Author(s) 2009. This article is published with open access at Springerlink.com

\begin{abstract}
Purpose This study was designed to retrospectively analyze outcomes of axillofemoral bypass (AxFB) operations performed in patients with severe comorbidities.

Methods All patients $(n=45)$ who received an AxFB between 1990 and 2005 for aortoiliac occlusive disease (AIOD, $n=35$ ) or infectious aortic disease (IAD, $n=10$ ) were included. Information on patency of the bypass and mortality was retrieved from patient records. A KaplanMeier survival analysis was performed to illustrate survival rates, limb salvage, and primary and secondary patency. Results Included patients had several comorbidities and a high operative risk. In this group, a 30-day mortality rate of $20 \%$ was found: $17 \%$ for the AIOD group, and $30 \%$ for the IAD group. During 5-year follow-up 20 patients died, of which 15 during the first year after operation. Survival rates were at 64 and $41 \%$ at 1 and 5 years and limb salvage rates were $84 \%$ for both these years. Primary patency rates at 1 and 5 years were 72 and 58\%, respectively, and secondary patency rates were $86 \%$ at both time points.

Conclusions High mortality rates were found in AIOD or IAD patients who received an AxFB. However, for high-
\end{abstract}

M. H. Liedenbaum

Department of Radiology, Academic Medical Center

Amsterdam, Amsterdam, The Netherlands

\section{F. J. Verdam ( $\square)$}

Department of General Surgery, University Hospital Maastricht,

Maastricht, The Netherlands

e-mail: froukjeverdam@yahoo.com

D. Spelt · H. G. W. de Groot · J. van der Waal · L. van der Laan Department of Surgery, Amphia Hospital, Breda, The Netherlands risk patients with an already reduced life expectancy, the AxFB remains an alternative with acceptable patency rates.

\section{Introduction}

The axillo(bi)femoral bypass (AxFB) operation is an alternative to direct arterial reconstruction, such as aortobifemoral grafting. This procedure is performed in patients with aortic graft sepsis or a mycotic aneurysm and in patients with a totally occluded abdominal aorta with a high operative risk. The advantage of the AxFB operation is that it is a less invasive operation compared with a total reconstruction of the aorta and that no surgical replacement of the infected aortobifemoral graft is required in the "hostile" abdomen.

Previous retrospective studies in groups varying in size from 34 to 108 patients who underwent surgery for aortoiliac occlusive disease (AIOD) or infectious aortic disease (IAD; infected aortic graft or mycotic aneurysm) reported 30-day mortality rates after AxFB surgery between 4 and 13\%. Primary patency rates at 5 years varied widely from 57 to $74 \%$ [1-6].

Compared with the AxFB, aortic bifurcation grafts for aortoiliac occlusive disease or for replacement of the infected aortic graft give higher survival rates, lower 30-day mortality, and appear to have better patency rates from previous studies [6-11]. Thirty-day mortality varies from 3.9 to $8 \%$ in these patients and the survival rate after 5 years is approximately $63-89 \%$. Furthermore, the primary patency after 5 years varies from 70 to $89 \%$. These outcomes are generally better than the reported outcomes for the AxFB in both AIOD and IAD patients, but in these patients with several comorbidities and a high operative risk aortic surgery is not preferable. The survival rates after 
operation in this complicated population mean that a less risky operation, such as the AxFB, might be a good alternative. Although several previous studies are performed in AxFB grafting, the current role of the AxFB for the management of AIOD and IAD in high-risk patients is not clear.

For this reason, this study was designed to investigate indications, risk factors, comorbidities, and outcomes of AxFB surgery for AIOD or IAD. Limb salvage, primary and secondary patency rates, and mortality of the AxFB operation were analyzed.

\section{Methods}

The medical records of patients who received an AxFB between 1990 and 2005 in the BLINDED (Amphia hospital, Breda, the Netherlands) were retrospectively analyzed. Information about cardiovascular risk factors, previous operations, and existing comorbidities was obtained from the patient records. Risk factors included smoking, diabetes, hyperlipidemia, hypertension, coronary artery disease, and cerebrovascular disease. Six staff vascular surgeons performed the AxFB operation under general anesthesia based on two different indications. Routinely, two vascular surgeons operated as a team to reduce the operation time.

The first indication for AxFB operation was aortoiliac occlusive disease (AIOD) in patients who had a high operative risk as a result of impaired function of one or more vital organs. The operative risk was classified according the ASA classification by an anesthesiologist [12]. Patients with recent myocardial infarction, congestive heart failure, significant anginal symptoms, chronic obstructive lung disease, or chronic renal insufficiency were candidates for axillofemoral grafting (ASA class III). The second indication was infectious aortic disease (IAD) in patients with an infected abdominal aorta or aortic graft sepsis.

All patients received duplex scanning and/or CT or catheter angiography preoperatively to evaluate the extent of lower extremity disease. Graft placement was done with a PTFE or Dacron bypass of 6- or 8-mm diameter.

Information on 30-day mortality rates, survival time, and graft patency was obtained from patient records. When possible, a follow-up of 5 years was performed in the outpatient clinic. The general practitioner from each patient who terminated outpatient clinic follow-up was contacted. The graft patency was evaluated by clinical examination, ankle-arm indices, and/or duplex scanning. Kaplan-Meier survival analysis was used to plot survival, limb salvage, and primary and secondary patency rates. Differences in survival rate and primary patency for graft type (Dacron or PTFE), graft size, and the site of anastomosis were calculated with the log-rank test.

\section{Results}

A total of 46 patients received an AxFB. One patient was excluded from analysis because the patient records were lost. Therefore, 45 patients remained for analysis: 29 men and 16 women. Eighteen patients received an axillounifemoral bypass, and 27 patients were treated with an axillobifemoral bypass. The median age of the total patient group was 70 (range 45-85) years. All patients had ASA class III or higher. In comparison, only $64 \%$ of patients who received an aortobifemoral bypass in our hospital had this ASA classification. Table 1 shows patient characteristics with cardiovascular risk factors, existing comorbidities, and previous operations. There were no significant differences in risk factors between AIOD and IAD patients.

A PTFE bypass was placed in 25 patients (of which 21 with AIOD), a Dacron bypass in 17 patients (13 with AIOD), and in 3 patients the type of bypass was unknown. To prevent graft occlusion, before 2000, all patients were treated after surgery with anticoagulation to reach an INR $>2.5$. After publication of the BOA trail, our hospital guidelines were adjusted and all patients were treated with antiplatelet therapy [13].

The 30-day mortality rate was $20 \%$ (9/45 patients). During 5 years of follow-up, 20 patients died and 15 of these patients died during the first year after operation. Figure 1 shows the Kaplan-Meier survival curves after 5-year follow-up, and Table 2 shows patients at risk and survival rates per interval. Follow-up varied from 1 to 60 (median 16) months. The graft occluded in 14 patients (31\%) during follow-up. Amputation was needed in six patients. The limb salvage rate at 1 year was $84 \%$ and at 5 years also was $84 \%$ (see Table 3 for limb salvage rates). Primary patency rates at 1,3 , and 5 years were 72,58 , and $58 \%$, respectively. The Kaplan-Meier curves of the primary patency are shown in Fig. 2, and the cumulative patency rates per interval are shown in Table 4 . The secondary patency at 1 year was $86 \%$ and remained equal in the following years (Fig. 3 and Table 5). Survival and patency were not different between patients with a Dacron or PTFE bypass $(P>0.05)$. Furthermore, the graft size did not influence survival $(P>0.05)$. However, primary patency of the 8-mm grafts was better than that of the 6 -mm grafts $(P<0.05)$. Primary patency after 1 year of follow-up in patients with 8 -mm grafts was $80 \%$ compared with $69 \%$ for the $6-\mathrm{mm}$ grafts.

In 22 patients the distal anastomosis of the bypass was made to the common femoral artery, in 9 patients to the superficial femoral, and in 9 patients to the profunda femoral artery. In five patients, the site of anastomosis was not stated in the operation files. The site of anastomosis and the preoperative patency of the femoral artery did not lead to different outcomes of primary patency $(P>0.05)$. 
Table 1 Patient characteristics

Data in parentheses are percentage of total number in group

COPD chronic obstructive pulmonary disease

${ }^{a}$ Malignancies that occurred during the period of $\mathrm{AxFB}$ operation: mamma carcinoma, colon carcinoma, larynx carcinoma and bronchus carcinoma

b This includes: aortoiliac or femoral bypass, femoropopliteal bypass, femorofemoral bypass and iliofemoral bypass

Fig. 1 In the left figure survival until 5 years is shown for the total group and in the right figure for the infectious aortic disease group (-) and the aortoiliac occlusive disease group (-). The censored points $(-$ and +$)$ are the patients who were lost during follow-up
Total $(N=45)$ Aortoiliac occlusive Infectious aortic disease $(n=35) \quad$ disease $(n=10)$

\begin{tabular}{lccc}
\hline & Total $(N=45)$ & $\begin{array}{l}\text { Aortoiliac occlusive } \\
\text { disease }(n=35)\end{array}$ & $\begin{array}{l}\text { Infectious aortic } \\
\text { disease }(n=10)\end{array}$ \\
\hline $\begin{array}{l}\text { Patient characteristics } \\
\text { Age in years (range) }\end{array}$ & $70(45-85)$ & $70(48-85)$ & $64(45-80)$ \\
Male & $29(64 \%)$ & $21(60 \%)$ & $8(80 \%)$ \\
Cardiovascular risk factors & & & \\
Smoking & $32(71 \%)$ & $24(77 \%)$ & $8(80 \%)$ \\
Hypertension & $23(51 \%)$ & $19(58 \%)$ & $4(40 \%)$ \\
$\begin{array}{l}\text { Diabetes } \\
\text { Coronary heart disease }\end{array}$ & $12(27 \%)$ & $10(29 \%)$ & $2(20 \%)$ \\
Hyperlipidemia & $16(36 \%)$ & $13(37 \%)$ & $3(30 \%)$ \\
Cerebrovascular disease & $19(42 \%)$ & $15(43 \%)$ & $4(40 \%)$ \\
Comorbidities & $14(31 \%)$ & $10(29 \%)$ & $4(40 \%)$ \\
Malignancy & & & \\
COPD & $4(9 \%)$ & $4(11 \%)$ & $1(10 \%)$ \\
Chronic renal insufficiency & $12(27 \%)$ & $11(31 \%)$ & $1(10 \%)$ \\
Previous operations & $3(7 \%)$ & $2(6 \%)$ & $2(20 \%)$ \\
Amputation of upper or lower leg & & & $4(40 \%)$ \\
Bypass operation for arterial occlusive disease $^{\mathrm{b}}$ & $14(31 \%)$ & $10(29 \%)$ &
\end{tabular}

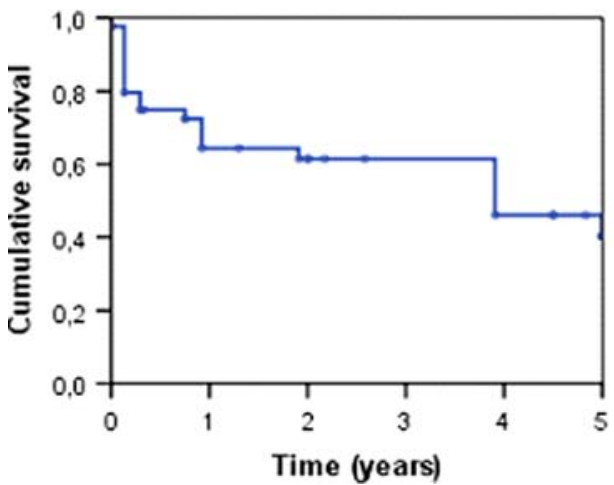

Hereafter, the results are presented separately for the two different indications.

\section{Aortoiliac occlusive disease (AIOD)}

This group consisted of 35 high-risk patients; 10 patients had an occluded abdominal aorta, 6 had a total occlusion of the iliac artery, 4 had a thrombus in the original aortobifemoral graft, and 15 had an infrarenal calcified aortic wall. The mean age of these patients was 70.2 years. Smoking was the most prevalent risk factor with $77 \%$ (see Table 1 for more patient characteristics).

The following indications for surgery in the AIOD group were described: 2 patients with claudication, 22 suffered from rest pain, 10 had established tissue loss, and for 1 patient the indication was not described. Median length of hospital stay was 18 (range, 9-65) days. Six patients $(17 \%)$ died within 1 month of operation. One patient died from a disseminated intravascular coagulation, which led to pulmonary emboli and gastrointestinal bleeding, three patients developed sepsis, and two patients died from a cerebrovascular accident. Figure 1 shows the Kaplan-Meier survival curves with a 5-year follow-up (see Table 2 for interval rates).

In total, the graft occluded in 12 patients $(34 \%)$ during follow-up. Two patients received a second AxF bypass because of an occlusion of the former AxF bypass, both due to a thrombosis of the subclavian artery. One patient received a femorofemoral crossover bypass. In six cases thrombectomy or urokinase treatment was performed to treat the occluded graft. Amputation took place in three patients as primary intervention after occlusion of the graft. An amputation after a primary intervention, e.g., lower leg amputation or thrombectomy, was performed in two patients in this group. Limb salvage was $83 \%$ after 1 and 5 years. Five patients underwent a secondary intervention for reocclusion. 
Table 2 Survival rates axillofemoral bypass for AIOD and IAD

Interval No. patients No. No. patients Cumulative SE (year) at risk deaths withdrawn ${ }^{\mathrm{a}}$ survival (\%) (\%)

All patients (both AIOD and IAD)

\begin{tabular}{lrrrrr}
$0-1$ & 45 & 15 & 7 & 64 & 7.5 \\
$1-2$ & 23 & 1 & 2 & 61 & 7.7 \\
$2-3$ & 20 & 2 & 4 & 54 & 8.4 \\
$3-4$ & 14 & 2 & 1 & 46 & 8.8 \\
$4-5$ & 11 & 0 & 3 & 46 & 8.8 \\
$A I O D$ & & & & & \\
$0-1$ & 35 & 11 & 7 & 65 & 8.6 \\
$1-2$ & 17 & 1 & 2 & 61 & 9.0 \\
$2-3$ & 14 & 2 & 3 & 50 & 10.2 \\
$3-4$ & 9 & 0 & 1 & 50 & 10.2 \\
$4-5$ & 8 & 0 & 2 & 50 & 10.2 \\
$I A D$ & & & & & \\
$0-1$ & 10 & 4 & 0 & 60 & 15.5 \\
$1-2$ & 6 & 0 & 0 & 60 & 15.5 \\
$2-3$ & 6 & 0 & 1 & 48 & 16.4 \\
$3-4$ & 5 & 2 & 0 & 36 & 16.1 \\
$4-5$ & 3 & 0 & 1 & 36 & 16.1 \\
\hline
\end{tabular}

${ }^{a}$ Patients were withdrawn because they were lost to follow-up

Table 3 Limb salvage rates of the axillofemoral bypass for both AIOD and IAD patients All patients (both AIOD and IAD)

\begin{tabular}{llllll}
\hline $\begin{array}{l}\text { Interval } \\
\text { (year) }\end{array}$ & $\begin{array}{l}\text { No. } \\
\text { patients } \\
\text { at risk }\end{array}$ & $\begin{array}{l}\text { No. } \\
\text { amputations }\end{array}$ & $\begin{array}{l}\text { No. patients } \\
\text { withdrawn }\end{array}$ & $\begin{array}{l}\text { Cumulative } \\
\text { limb salvage } \\
(\%)\end{array}$ & $\begin{array}{l}\text { SE } \\
(\%)\end{array}$ \\
\hline $0-1$ & 45 & 6 & 17 & 84 & 6.0 \\
$1-2$ & 22 & 0 & 3 & 84 & 6.0 \\
$2-3$ & 19 & 0 & 5 & 84 & 6.0 \\
$3-4$ & 14 & 0 & 3 & 84 & 6.0 \\
$4-5$ & 11 & 0 & 0 & 84 & 6.0 \\
\hline
\end{tabular}

${ }^{a}$ Patients were withdrawn because of death or were lost to follow-up. Rates for the AIOD group and IAD group are not given separately, but numbers are mentioned in the text
Primary patency rates in the aortoiliac occlusive group at 1,3 , and 5 years were 69,51 , and $51 \%$ respectively. The Kaplan-Meier curves of the primary patency are shown in Fig. 2 (see Table 4 for interval patency rates). The secondary patency at 1 year was $86 \%$ and remained equal in the following years (Fig. 3).

Infectious aortic disease (IAD)

The 10 patients with IAD had a median age of 64 years. Median length of hospital stay was 42 (range, 2-99) days. Most patients had multiple risk factors (Table 1). Three patients (30\%) died within 1 month of operation: one patient died due to multiple organ failure, one patient had a cardiac arrest, and one appeared to die from rupture of the aortic stump. No autopsy was performed in this last patient. The Kaplan-Meier survival curves for this patient group are shown in Fig. 1.

During the first year, two bypasses obstructed, which led to thrombectomy, but in the following years none of the other bypasses obstructed. The primary patency rate at 1,3 , and 5 years was therefore $80 \%$ (Fig. 2). Only one of the two bypasses that was obstructed occluded again within a few days, resulting in a secondary patency of $90 \%$ during 5 years of follow-up. This bypass reocclusion resulted in an amputation. Limb salvage at 1 year was $90 \%$. None of the patients had an infection of the AxFB graft after their operation.

\section{Discussion}

In the literature various outcomes in patency and mortality rates of the axillofemoral bypass have been reported. In this study we evaluated the outcomes of high-risk patients who received an AxFB in our hospital for aortoiliac occlusive disease (AIOD) or infectious aortic disease (IAD). For the whole population, our results showed a high 30 -day mortality rate of $20 \%$ and acceptable patency rates:
Fig. 2 In the left figure primary patency until 5 years is shown for the total group and in the right figure for the infectious aortic disease group (-) and the aortoiliac occlusive disease group (-). The censored points $(-$ and +$)$ are the patients who were lost during follow-up
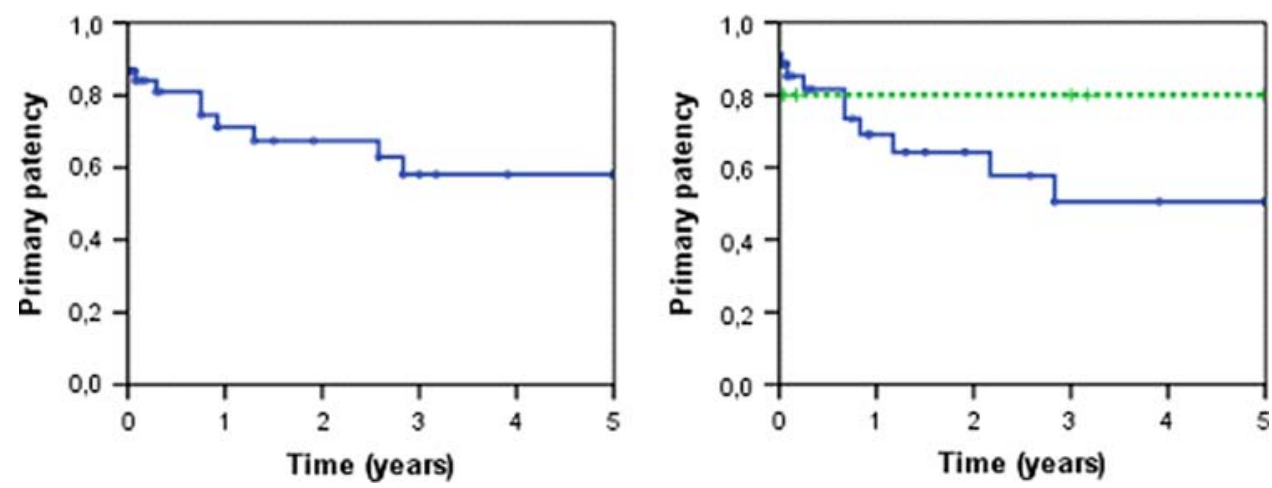
Table 4 Primary patency axillofemoral bypass for AIOD and IAD

\begin{tabular}{llllll}
\hline $\begin{array}{l}\text { Interval } \\
\text { (year) }\end{array}$ & $\begin{array}{l}\text { No. } \\
\text { grafts at } \\
\text { risk }\end{array}$ & $\begin{array}{l}\text { No. } \\
\text { occluded } \\
\text { grafts }\end{array}$ & $\begin{array}{l}\text { No. patients } \\
\text { withdrawn }^{\mathrm{a}}\end{array}$ & $\begin{array}{l}\text { Cumulative } \\
\text { patency }(\%)\end{array}$ & $\begin{array}{l}\text { SE } \\
(\%)\end{array}$ \\
\hline
\end{tabular}

All patients (both AIOD and IAD)

\begin{tabular}{lrrrrr}
$0-1$ & 45 & 11 & 16 & 72 & 7.6 \\
$1-2$ & 18 & 1 & 2 & 68 & 8.1 \\
$2-3$ & 15 & 2 & 2 & 58 & 9.5 \\
$3-4$ & 11 & 0 & 3 & 58 & 9.5 \\
$4-5$ & 8 & 0 & 0 & 58 & 9.5 \\
AIOD & & & & & \\
$0-1$ & 35 & 9 & 13 & 69 & 8.9 \\
$1-2$ & 13 & 1 & 2 & 64 & 9.7 \\
$2-3$ & 10 & 2 & 2 & 49 & 12 \\
$3-4$ & 6 & 0 & 1 & 49 & 12 \\
$4-5$ & 5 & 0 & 0 & 49 & 12 \\
$I A D$ & & & & & \\
$0-1$ & 10 & 2 & 3 & 80 & 12.6 \\
$1-2$ & 5 & 0 & 0 & 80 & 12.6 \\
$2-3$ & 5 & 0 & 0 & 80 & 12.6 \\
$3-4$ & 5 & 0 & 2 & 80 & 12.6 \\
$4-5$ & 3 & 0 & 0 & 80 & 12.6 \\
\hline
\end{tabular}

$\overline{{ }^{a} \text { Patients were withdrawn because of death or were lost to follow-up }}$

$72 \%$ for primary patency and $86 \%$ for secondary patency at 1 year follow-up.

Considering only the AIOD group, we found a mortality rate of $17 \%$. This is much higher than in a similar study by Martin and Katz where in 60 patients the 30-day mortality rate was only $4.9 \%$. The mean age of this group was 74 years and there were comparable percentages of comorbidities, e.g., diabetes and smoking [5]. Schneider et al. describe a 30-day mortality rate of 9\% (3/34 patients) in their AxFB group, but during hospitalization three other patients died in this study, resulting in a hospital mortality rate of $18 \%$ [14]. This was relatively high compared with the aortobifemoral bypass group in this same study with a mortality rate of $1 \%$ during hospitalization. It must be considered, however, that the patients in the AxFB group
Table 5 Secondary patency axillofemoral bypass for AIOD and IAD

\begin{tabular}{llllll}
\hline $\begin{array}{l}\text { Interval } \\
\text { (year) }\end{array}$ & $\begin{array}{l}\text { No. } \\
\text { grafts at } \\
\text { risk }\end{array}$ & $\begin{array}{l}\text { No. } \\
\text { occluded } \\
\text { grafts }\end{array}$ & $\begin{array}{l}\text { No. patients } \\
\text { withdrawn }^{\mathrm{a}}\end{array}$ & $\begin{array}{l}\text { Cumulative } \\
\text { patency }(\%)\end{array}$ & $\begin{array}{l}\text { SE } \\
(\%)\end{array}$ \\
\hline
\end{tabular}

All patients (both AIOD and IAD)

\begin{tabular}{rrrrrr}
$0-1$ & 45 & 5 & 20 & 86 & 6.1 \\
$1-2$ & 20 & 0 & 3 & 86 & 6.1 \\
$2-3$ & 17 & 0 & 4 & 86 & 6.1 \\
$3-4$ & 13 & 0 & 3 & 86 & 6.1 \\
$4-5$ & 10 & 0 & 2 & 86 & 6.1 \\
\hline
\end{tabular}

${ }^{\text {a }}$ Patients were withdrawn because of death or were lost to follow-up Rates for the AIOD group and IAD group are not given separately, but numbers are mentioned in the text

were older and had more major risk factors than the aortobifemoral bypass patients in this study. Therefore, the difference in mortality rates in AxFB studies might be due to the differences in comorbidities and the patients' physical condition preoperatively. Patients with more comorbidities have a lower life expectancy and have less chance of survival after operation. The median age of AIOD patients in our study was 71 and many had comorbidities, indicating that these patients have a low life expectancy, which may explain the high mortality rate after operation in this population. The hospital stay was long in both patient groups in our study, because almost half of the patients had postoperative complications, such as wound infections, urine tract infections complicated with sepsis, pneumonia, and neurologic complaints.

Regarding graft patency, somewhat different outcomes were found in previous studies. We found a primary patency rate of $49 \%$ at 3 years for the AIOD patients, which was $72 \%$ in the study by Martin and Katz and 63\% by Schneider et al. [5, 14]. In our study two patients $(6 \%)$ received a second AxFB graft because of occlusion of the primary graft. Both of these secondary AxFB grafts remained patent, but in nearly all patients with graft failure who were treated with urokinase, thrombectomy, or amputation in this study, the graft did not remain patent
Fig. 3 In the left figure secondary patency until 5 years is shown for the total group and in the right figure for the infectious aortic disease group (-) and the aortoiliac occlusive disease group (-). The censored points $(-$ and +$)$ are the patients who were lost during follow-up
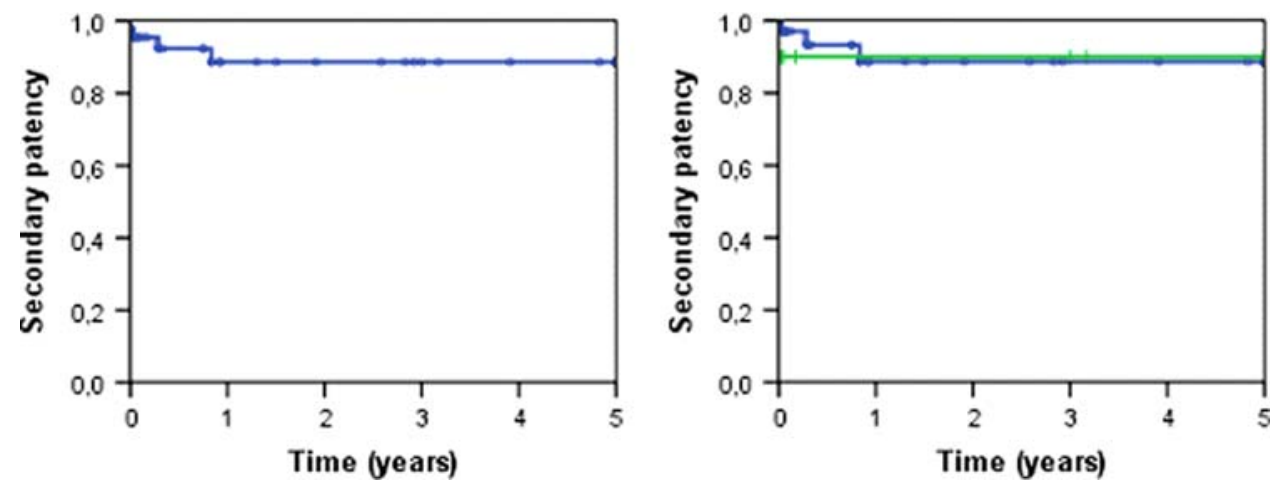
after treatment and a secondary intervention was necessary. Olson et al. examined the results of repeat AxFB grafting as treatment for graft failure. They found that AxFB replacement and/or anastomotic revision had a superior patency to thrombectomy. At 18 months mean patency after thrombectomy was $11 \%$, whereas patency for graft replacement was 54\% [15]. These rates seem to be consistent with our results. We, therefore, think that AxFB replacement, especially after a proximal occlusion (e.g., stenosis of the subclavian artery), should be the first procedure of choice.

Considering the results of AxFB grafting in the infectious aortic disease group, we found different outcomes than several earlier published studies in similar AxFBgrafted patients. The infectious 30-day mortality rate in the infectious aortic disease group in our study was high (30\%). Previously a postoperative mortality rate of $13 \%$ was found in patients who received an AxFB after removal of the infected aortic graft [2]. Comparable postoperative mortality rates (11-15\%) were found for treatment of aortic graft infection with extra-anatomic bypass grafting $[1,16]$. Another treatment option for aortic graft infection is excision of the infected aortic graft and a simultaneous placement of a new aortic graft. There are several types of grafts available, such as a vein autograft or a prosthetic graft. In studies where those graft replacements were studied, the 30-day mortality varied from 8 to $20 \%$ and the 5 -year primary patency from 66 to $91 \%$ [7, 17, 18].

Furthermore, Johnson et al. found that the use of different types of grafts for the AxFB operation results in similar long-term patencies [19]. Although in this study we did not find a significant difference in outcomes of patency between both types of grafts, when comparing the graft size, we found a significantly better primary patency for the largest size ( $8 \mathrm{~mm}$ vs. $6 \mathrm{~mm}$ ). This difference might be due to an increased wall sheer stress in the smaller diameter graft, which could increase the probability to develop stenosis [20]. Therefore, we now prefer to use the 8-mm graft for AxFB operations.

A limitation of this study was the long time period (1990-2005) in which patients were included and followed. This raises the possibility of a change in practice patterns and variability due to advances in perioperative care during the study period. We were, however, not able to identify those changes and thus to analyze their influence. Because of the relatively poor outcomes of AxFB compared with other operations, such as an aortobifemoral bypass, we adjusted the management of operating high-risk patients in our hospital. In recent years only high-risk patients with a very short life expectancy $(<5$ years) are treated with an AxFB. This is contrary to an increase in abdominal endovascular therapy in our hospital; during the period from 1990 to 2000, 150 endovascular procedures were performed yearly, and from 2000 more than 200 procedures yearly. In our practice, endovascular therapy is now the preferred option for treatment of occlusive arterial disease. The AxFB is applied only in specific cases in which endovascular therapy is not possible.

Another limitation of this study is that it was based on retrospective research and clinical records, which are not always complete. A prospective study with closer attendance to follow-up might have gathered more information on factors predictive of graft failure, including information on the patency of the superficial femoral arteries and the pre- and postoperative ankle-arm indices.

\section{Conclusions}

The axillofemoral bypass operation has relatively high postoperative mortality rates but acceptable patency outcomes in our study. The high mortality rates are partially explained by an already short life expectancy of patients. In patients with a reasonable life expectancy alternative treatment, such as aortofemoral bypass for aortoiliac occlusive disease or autogenous graft for infectious aortic disease, may be preferred. However, for high-risk patients with a reduced life expectancy, axillofemoral bypass is still an acceptable alternative for treatment of aortoiliac occlusive disease or infectious aortic disease.

Acknowledgments The authors thank Roos Met for critical review of this manuscript.

Open Access This article is distributed under the terms of the Creative Commons Attribution Noncommercial License which permits any noncommercial use, distribution, and reproduction in any medium, provided the original author(s) and source are credited.

\section{References}

1. Seeger JM, Pretus HA, Welborn MB et al (2000) Long-term outcome after treatment of aortic graft infection with staged extra-anatomic bypass grafting and aortic graft removal. J Vasc Surg 32:451-459

2. Yeager RA, Taylor LM Jr, Moneta GL et al (1999) Improved results with conventional management of infrarenal aortic infection. J Vasc Surg 30:76-83

3. Jamsen T, Tulla H, Loponen P (1999) Axillofemoral bypass operations in Kuopio University Hospital 1985-1996. Ann Chir Gynaecol 88:269-275

4. Passman MA, Taylor LM, Moneta GL, Edwards JM, Yeager RA, McConnell DB, Porter JM (1996) Comparison of axillofemoral and aortofemoral bypass for aortoiliac occlusive disease. J Vasc Surg 23:263-271

5. Martin D, Katz SG (2000) Axillofemoral bypass for aortoiliac occlusive disease. Am J Surg 180:100-103

6. Onohara T, Komori K, Kume M et al (2000) Multivariate analysis of long-term results after an axillobifemoral and aortobifemoral 
bypass in patients with aortoiliac occlusive disease. J Cardiovasc Surg (Torino) 41:905-910

7. Young RM, Cherry KJ, Davis PM, Gloviczki P, Bower TC, Panneton JM, Hallett JW (1999) The results of in situ prosthetic replacement for infected aortic grafts. Am J Surg 178:136-140

8. Nevelsteen A, Wouters L, Suy R (1991) Aortofemoral Dacron reconstruction for aorto-iliac occlusive disease: a 25 -year survey. Eur J Vasc Surg 5:179-186

9. Oderich GS, Bower TC, Cherry KJ, Panneton JM, Sullivan TM, Noel AA, Carmo M, Cha S, Kalra M, Gloviczki P (2006) Evolution from axillofemoral to in situ prosthetic reconstruction for the treatment of aortic graft infections at a single center. $\mathrm{J}$ Vasc Surg 43:1166-1174

10. Davidovic L, Vasic D, Maksimovic R, Kostic D, Markovic D, Markovic M (2004) Aortobifemoral grafting: factors influencing long-term results. Vascular 12:171-178

11. De Vries SO, Hunnink MG (1997) Results of aortic bifurcation grafts for aortoiliac occlusive disease: a meta-analysis. J Vasc Surg 26:558-569

12. Wolters U, Wolf T, Stützer H et al (1996) ASA classification and perioperative variables as predictors of postoperative outcome. $\mathrm{Br}$ J Anesth 77:217-222

13. BOA Study Group (2000) Efficacy of oral anticoagulants compared with aspirin after infrainguinal bypass surgery (The Dutch
Bypass Oral anticoagulants or Aspirin study): a randomised trial. Lancet 355(9201):346-351

14. Schneider JR, McDaniel MD, Walsh DB et al (1992) Axillofemoral bypass: outcome and hemodynamic results in high-risk patients. J Vasc Surg 15:952-962

15. Olson CJ, Edwards JM, Taylor LM et al (2002) Repeat axillofemoral grafting as treatment for axillofemoral graft occlusion. Arch Surg 137:1364-1367

16. Schmitt DD, Seabrook GR, Bandyk DF, Towne JBJ (1990) Graft excision and extra-anatomic revascularization: the treatment of choice for the septic aortic prosthesis. Cardiovasc Surg (Torino) 31:327-332

17. Daenens K, Fourneau I, Nevelsteen A (2003) Ten-year experience in autogenous reconstruction with the femoral vein in the treatment of aortofemoral prosthetic infection. Eur J Vasc Endovasc Surg 25:240-245

18. Kieffer E, Gomes D, Chiche L et al (2004) Allograft replacement for infrarenal aortic graft infection: early and late results in 179 patients. J Vasc Surg 39:1009-1017

19. Johnson WC, Lee KK (1999) Comparative evaluation of externally supported Dacron and polytetrafluoroethylene prosthetic bypasses for femorofemoral and axillofemoral arterial reconstructions. J Vasc Surg 30:1077-1083

20. Qiao A, Liu Y, Guo Z (2006) Wall shear stresses in small and large two-way bypass grafts. Med Eng Phys 28:251-258 